Copyright (C) 2014 by Academic Publishing House Researcher

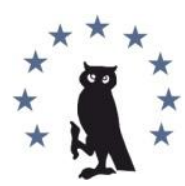

Published in the Russian Federation

European Researcher

Has been issued since 2010.

ISSN 2219-8229

E-ISSN 2224-0136

Vol. 83, No. 9-2, pp. 1648-1650, 2014

DOI: $10.13187 /$ er.2014.83.1648

www.erjournal.ru

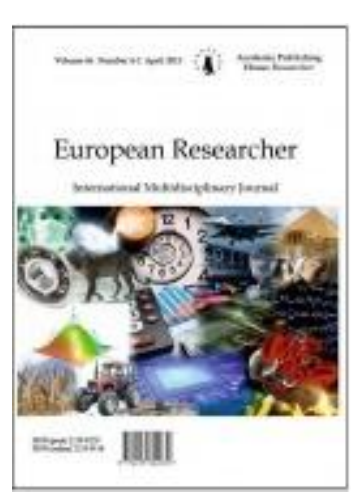

Physical and Mathematical sciences

Физико-Математические науки

\title{
Use of Resolving Equation to Define the Lower Critical Reynolds Number
}

\author{
${ }^{1}$ Alexander A. Solovyev \\ ${ }^{2}$ Constantin V. Chekarev
}

1-2 Lomonosov Moscow State University, Russian Federation

${ }^{1}$ Doctor of Physical and Mathematical sciences, Professor

${ }^{2}$ Researcher

E-mail: geosolmgu@mail.ru

\begin{abstract}
Although the issue of streams with non-crossing trajectories of particle motions ranging from chaotic, random with irregular current lines, has been given a lot of attention, it still remains unresolved. The study features a relevant issue for hydromechanics, which is precise values determination of the Lower Critical Reynolds Number. It is suggested to put forward an updated approach to the use of energetic analysis for analytical calculation of the Reynolds Resolving Equation. The assessment of transition to mean motion from pulsation to the direction of laminar flows was fulfilled.

Keywords: turbulence energy; resolving equation; the Lower Critical Reynolds Number.

\section{Introduction}

The study features an illustration of researched steady direct motion of incompressible viscous fluid in the direction $\mathrm{X}$ caused by the pressure between two parallel walls $\left(\mathrm{y}= \pm \mathrm{b}_{\mathrm{o}}\right.$.). On the walls, the conditions for the adhesion and the equality to zero of the derivatives of velocity for all coordinates are created. Conditions are created for the pulsating motion of periodicity of a perturbation for the coordinate $\mathrm{x}$ with the period $\lambda$ and the condition of the symmetry of the turbulent stress.
\end{abstract}

\section{Definition}

As a result of integration of the energy equation for Reynolds relative molar motion [3] the discriminating equation that defines the energy transition of the relative molar motion to the energy of the mean molar motion and heat has following form:

$$
-v \int_{-b_{o}}^{b_{o}}\left[\ell^{4}\left(\alpha^{2}+\beta^{2}\right)+2 \ell^{2}\left(\dot{\alpha}^{2}+\dot{\beta}^{2}\right)+\ddot{\alpha}^{2}+\ddot{\beta}^{2}\right] d y=\ell \int_{-b_{o}}^{b_{o}} \frac{d \bar{V}}{d y}(\alpha \dot{\beta}-\beta \dot{\alpha}) d y,
$$


where $\alpha, \beta$, dots over symbols denote derivatives for $y, \ell=2 \pi / \lambda$, - the wave number of pulsations, $-V$ kinematics coefficient of viscosity. Included in the formula (1) the derivative of the mean velocity $\bar{V}$ in the direction across the flow, $\frac{d \bar{V}}{d y}$.in contrast to Reynolds [3] is determined not from the Poiseuille law for laminar flows, but from the turbulent profile of velocity distribution [4]

$$
\frac{\mathrm{U}_{\max }-\overline{\mathrm{V}}}{\mathrm{V}_{*}}=2,5 \ln \mathrm{b}_{\mathrm{o}} / \mathrm{y}
$$

Integrating by parts the right side of the discriminating equation (1) and taking into account the conditions of the symmetry of pulsation we obtain the discriminating equation in the following form:

$$
\frac{5 \mathrm{~V}_{*} \mathrm{~b}_{\mathrm{o}}}{\mathrm{v}}=\frac{\mathrm{E}_{2}}{\mathrm{E}_{1}} .
$$

where $\mathrm{E}_{1}$ - the transition energy of the mean molar motion in heat is defined by the equation :

$$
\mathrm{E}_{1}=\frac{1}{2 \mathrm{~b}_{\mathrm{o}}} \int_{-\mathrm{b}_{\mathrm{o}}}^{\mathrm{b}_{\mathrm{o}}} \frac{\mathrm{dy}}{\mathrm{y}^{2}} \int_{-\mathrm{b}_{\mathrm{o}}}^{\mathrm{y}} \ell(\beta \dot{\alpha}-\alpha \dot{\beta}) \mathrm{dy},
$$

$\mathrm{E}_{2}$ - the energy of transition from mean molar motion to the relative molar is defined by the equation:

$$
\mathrm{E}_{2}=\int_{-\mathrm{b}_{\mathrm{o}}}^{\mathrm{b}_{\mathrm{o}}}\left[\ell^{4}\left(\alpha^{2}+\beta^{2}\right)+2 \ell^{2}\left(\dot{\alpha}^{2}+\dot{\beta}^{2}\right)+\ddot{\alpha}^{2}+\ddot{\beta}^{2}\right] \mathrm{dy} .
$$

In accordance with the boundary conditions the amplitudes of the pulsations can be written as fallowing:

$$
\alpha=a \cdot \sin p+a \cdot \sin 3 p, \quad \beta=a \cdot \sin 2 p+\frac{a}{2} \cdot \sin 4 p,
$$

where $p=\frac{\pi y}{2 b_{0}}$.

\section{Result}

As a result of integration of the equations (3) and (4) we obtain:

$$
\begin{gathered}
\mathrm{E}_{1}=\frac{\pi \mathrm{a}^{2} \mathrm{~L}}{16 \mathrm{~b}_{\mathrm{o}}^{3}}\left[3 \mathrm{Si}\left(\frac{\pi}{2}\right)+3 \operatorname{Si}\left(\frac{3 \pi}{2}\right)-\operatorname{Si}\left(\frac{5 \pi}{2}\right)-\operatorname{Si}\left(\frac{7 \pi}{2}\right)\right], \\
\mathrm{E}_{2}=\frac{\mathrm{a}^{2}}{\mathrm{~b}_{\mathrm{o}}^{3}}\left(\frac{\pi}{2}\right)^{4}\left[3,25 \mathrm{~L}^{4}+36 \mathrm{~L}^{2}+162\right] .
\end{gathered}
$$

where $\mathrm{L}=\frac{2 \mathrm{~b}_{\mathrm{o}}}{\pi} \ell$.

After substitution in the discriminating equation (2) of expressions (5), (6) and known from [4] relations of transition from dynamic velocity $V_{*}$ to mean velocity $\overline{\mathrm{V}}$ we obtain: 


$$
\operatorname{Re}=\frac{2 \mathrm{~b}_{\mathrm{o}} \overline{\mathrm{V}}}{v}=\frac{37,4\left(\mathrm{~L}^{4}+11 \mathrm{~L}^{2}+50\right)}{\mathrm{L}}
$$

\section{Conclusion}

The minimum of the expression (7) is determined by the value $L_{\min }=1,62$, of which gives $\mathrm{Re}_{\min }=2000$. This means that for the reverse flow transition from turbulent to laminar the discriminating equation, defining the low value of the critical Reynolds number is expressed through the pulsation frequency and the dimension of a stream near the wall surface. Similar calculations can be made for other types of flow.

\section{References:}

1. Liepmann H.W. (1979) The Rise and Fall of Ideas in Turbulence Amer. Sci. 67, 2. 221-228.

2. Jackson D., Launder B. (2007) Osborne Reynolds and the publication of his papers on turbulent flow. Annual. Review of Fluid Mechanics. 39. 19-35.

3 . Reynolds O. (1895) On the dynamical theory of incompressible viscous fluids and the determination of the criterion. Philosophical Transactions of the Royal Society of London. A. 186, 123-164.

4. Nigmatulin R.I., Solovyev A.A. (2012) Fundamentals of fluid mechanics. Moscow: Littera, 400 p. (in Russian) 